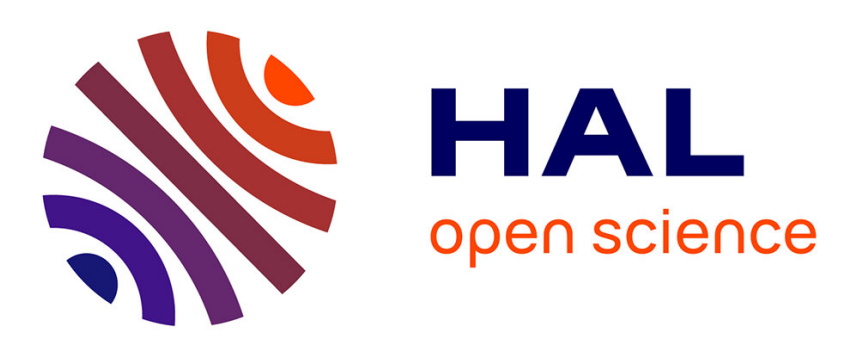

\title{
Lattice Boltzmann method for miscible gases: A forcing-term approach
}

\author{
Lucien Vienne, Simon Marié, Francesco Grasso
}

\section{To cite this version:}

Lucien Vienne, Simon Marié, Francesco Grasso. Lattice Boltzmann method for miscible gases: A forcing-term approach. Physical Review E , 2019, 100 (2), 10.1103/PhysRevE.100.023309 . hal$03091042 \mathrm{v} 2$

\section{HAL Id: hal-03091042 \\ https://hal.science/hal-03091042v2}

Submitted on 29 Aug 2019

HAL is a multi-disciplinary open access archive for the deposit and dissemination of scientific research documents, whether they are published or not. The documents may come from teaching and research institutions in France or abroad, or from public or private research centers.
L'archive ouverte pluridisciplinaire HAL, est destinée au dépôt et à la diffusion de documents scientifiques de niveau recherche, publiés ou non, émanant des établissements d'enseignement et de recherche français ou étrangers, des laboratoires publics ou privés. 


\title{
Lattice Boltzmann method for miscible gases: A forcing-term approach
}

\author{
Lucien Vienne $\odot,{ }^{*}$ Simon Marié, ${ }^{\dagger}$ and Francesco Grasso ${ }^{\ddagger}$ \\ DynFluid Laboratory, Conservatoire National des Arts et Métiers, 151 boulevard de l'hôpital, 75013 Paris, France
}

(Received 4 April 2019; revised manuscript received 8 July 2019; published 28 August 2019)

\begin{abstract}
A lattice Boltzmann method for miscible gases is presented. In this model, the standard lattice Boltzmann method is employed for each species composing the mixture. Diffusion interaction among species is taken into account by means of a force derived from kinetic theory of gases. Transport coefficients expressions are recovered from the kinetic theory. Species with dissimilar molar masses are simulated by also introducing a force. Finally, mixing dynamics is recovered as shown in different applications: an equimolar counterdiffusion case, Loschmidt's tube experiment, and an opposed jets flow simulation. Since collision is not altered, the present method can easily be introduced in any other lattice Boltzmann algorithms.
\end{abstract}

DOI: 10.1103/PhysRevE.100.023309

\section{INTRODUCTION}

This paper deals with the application of the lattice Boltzmann method (LBM) to mixing dynamics. Mixing occurs in many natural and industrial processes such as thermohaline circulation, pollute dispersion, diffusion in porous media, combustion, chemical processing, or mass and momentum transport in multicomponent fluids. An accurate understanding of mixing dynamics is of great importance for these applications.

In the simplest case of the mixing not affecting the flow dynamics, as is the case of the mixing of a dye or a tracing species, the Navier-Stokes or the lattice Boltzmann equations are solved to describe the flow dynamics, while the dye or species concentrations are modeled separately by simple convection-diffusion equations (passive scalar approach). However, mixing is often much more complex. The flow dynamics and the mixing process are heavily coupled and cannot be separated since mixing produces changes to the fluid. Mixing dynamics are then incredibly complicated as the interactions between various species need to be accounted for (e.g., collisions in the kinetic formulation). Whereas a large number of studies about global mixing dynamics are available, a good understanding of the microscopical processes involved in complex chemical mixing is still lacking.

The classical approach for the simulation of mixtures is based on the single-fluid approach that assumes as unknowns the species densities and the mixture velocity. The NavierStokes equations are solved for the mixture using phenomenological laws such as Fick's law or the Maxwell-Stefan equations for the species mass fluxes. This approach is often used in combustion with detailed chemistry, which involves a large number of species (see Ref. [1] for a lattice Boltzmann singlefluid model).

\footnotetext{
*Corresponding author: contact@1vienne.com

†simon.marie@lecnam.net

†rancesco.grasso@lecnam.net
}

However, the use of a mixture velocity as a unique unknown can lead to errors in the description of the flow dynamics. This is particularly true when the chemical properties of each species differ greatly and in situations where the mixing process depends on the chemical composition and on the velocities of each species. For instance, consider the case of two different coflowing gases separated by a splitter plate. On either side of the plate, the dynamics is governed by Navier-Stokes equations and a velocity is defined for each gas. Past the splitter plate, the two gases start mixing. In the case of the single-fluid approach, only one velocity is specified for the mixture, which may be defined in terms of either a mass, molar, or any other averages. Instead, in order to accurately depict the transient mixing dynamics, a more natural way is to consider the species densities and the velocities of each species as unknowns (as prior to mixing). This is the so-called multifluid approach.

The LBM provides an alternative and convenient way to model fluid flows compared to conventional macroscopic approaches [2]. Indeed, the algorithm is simple, computationally efficient due to its explicit formulation, and easily adapted for parallel computing. The LBM is therefore particularly appealing for the simulation of miscible mixtures. In the multifluid strategy, one introduces a distribution function for each species. In single-species flows, the collision of particles is approximated by the Bhatnagar-Gross-Krook (BGK) collision operator [3] and the distributions relax to equilibrium values at a rate that depends on the relaxation time. In the case of a mixture, there is no unique BGK formulation. Hence, different LBMs for multicomponent flows have been developed depending on the underlying kinetic theory of the mixture being investigated. One possible approach is to split collisions between molecules of the same species (self-collision) and collisions between molecules of different species (crosscollision). Luo and Girimaji [4] employ a linear collision based on Sirovich's kinetic theory of mixtures [5]. This work is further extended from binary to multicomponent flows in Refs. [6,7]. In his early work, Asinari [8,9] uses a model derived from Hamel's kinetic theory [10]. Other noticeable 
split collision models can be found in Refs. [11] and [12]. The latter authors exploit a fast-slow decomposition (quasiequilibrium to equilibrium) and is further extended in Ref. [13] to thermal multicomponent flows. Other approaches are based on a single global collision term such as Asinari's model [14], derived from the AAP-BGK collision operator [15]. The equilibrium velocity is given by the conservation of species momenta. Diffusive and viscous effects are separated in the momentum space. References [16-18] have slightly modified the formulation of the equilibrium state, and the definition of the equilibrium velocity and applications to electrolytes are reported in Ref. [19].

In contrast to the previous LBMs, the coupling terms due to diffusion are introduced in our model by means of a force derived from the kinetic theory of gases extended to mixtures $[20,21]$. We have extended the work of Kerkhof and Geboers [21] to formulate the forcing term in the framework of the LBM. Furthermore, the transport coefficients are derived from the kinetic theory of gases, and a forcingterm strategy is proposed to take into account species having dissimilar molecular masses. This latter force, the diffusion force, and the formulation of the transport coefficients constitute the three building blocks of the present model. One major advantage of the proposed method is the easiness of implementation. Since collision is not modified, the method can be introduced in any other lattice Boltzmann algorithms (e.g., Refs. [22-25]) to take into account complex diffusion among species.

This paper is laid out as follows. In Sec. II we introduce our simplified kinetic model for miscible gases. The macroscopic equations as well as the transport coefficients are presented in Sec. III. In Sec. IV we address the problem of the simulation of species with different molecular masses. Finally, in Sec. V we validate the main features of our model.

\section{A SIMPLIFIED KINETIC MODEL FOR MULTICOMPONENT MIXTURES}

The LBM is an alternative method for simulating fluid flows by solving a simplified formulation of the kinetic model. In this section, we present a LBM for mixtures of miscible gases. The model is an extension of the macroscopic theory of Kerkhof and Geboers [21], which is based on the work of Hirschfelder, Curtiss, and Bird on the kinetic theory of gases [20]. The model satisfies the indifferentiability principle, namely, for a mixture of like gases, it reduces to a singlespecies BGK model. In addition, the macroscopic MaxwellStefan equations are recovered for purely diffusive flows when convection is negligible.

A mixture is composed of multiple species, and each species is defined by its own distribution function, which is governed by its own kinetic equation. For the sake of simplicity we consider only a BGK-like collision operator. More advanced collisions operators such as multiple relaxation time, entropic, regularized, or cumulant operators, mostly developed to remedy some stability defects, could also be implemented [22-25]. Let $m$ and $n$ denote different species $(m, n=1,2, \ldots, N, N$ being the total number of species). The distribution function of species $m, f_{\alpha}^{m}$, obeys the following discrete kinetic equation:

$$
\begin{aligned}
f_{\alpha}^{m}\left(\mathbf{x}+\mathbf{e}_{\alpha} \delta_{t}, t+\delta_{t}\right)= & f_{\alpha}^{m}(\mathbf{x}, t)-\frac{\delta_{t}}{\tau_{m}}\left[f_{\alpha}^{m}(\mathbf{x}, t)-f_{\alpha}^{m(e q)}(\mathbf{x}, t)\right] \\
& +\delta_{t} S_{\alpha}^{m}(\mathbf{x}, t),
\end{aligned}
$$

where $\mathbf{x}, t, \alpha$, and $\tau_{m}$ are, respectively, the spatial coordinate, time, number of discrete kinetic velocities $\mathbf{e}_{\alpha}$, and relaxation time of each species. The equilibrium distribution functions, $f_{\alpha}^{m(e q)}$, are given by the standard polynomial formulation

$$
f_{\alpha}^{m(e q)}=\rho_{m} \omega_{\alpha}\left[1+\frac{\mathbf{u}_{m} \cdot \mathbf{e}_{\alpha}}{c_{s}^{2}}+\frac{\left(\mathbf{u}_{m} \cdot \mathbf{e}_{\alpha}\right)^{2}}{2 c_{s}^{4}}-\frac{\mathbf{u}_{m} \cdot \mathbf{u}_{m}}{2 c_{s}^{2}}\right] .
$$

$S_{\alpha}^{m}$ is the source term from Guo's forcing scheme [26], widely used in order to include forces in the lattice Boltzmann algorithm,

$$
S_{\alpha}^{m}=\left(1-\frac{\delta_{t}}{2 \tau_{m}}\right) \omega_{\alpha}\left[\frac{\mathbf{e}_{\alpha}-\mathbf{u}_{m}}{c_{s}^{2}}+\frac{\left(\mathbf{e}_{\alpha} \cdot \mathbf{u}_{m}\right) \mathbf{e}_{\alpha}}{c_{s}^{4}}\right] \cdot \mathcal{F}_{m},
$$

where $\mathcal{F}_{m}$ is the force acting on the $m$ th species, which is derived in the following to take into account the species interactions.

In this study, we use the so-called D2Q9 isothermal, two-dimensional, and nine-velocity discretization. Extension to the three-dimensional formulation (D3Q19 or D3Q27) is straightforward. The pseudosound velocity is $c_{s}^{2}=\frac{1}{3}$, the kinetic velocities are expressed as

$\mathbf{e}_{\alpha}=\left[\begin{array}{ccccccccc}0 & 1 & 0 & -1 & 0 & 1 & -1 & -1 & 1 \\ 0 & 0 & 1 & 0 & -1 & 1 & 1 & -1 & -1\end{array}\right]^{T} 1 \leqslant \alpha \leqslant 9$,

and the lattice weights are equal to

$$
\omega_{\alpha}=\left[\begin{array}{lllllllll}
\frac{4}{9} & \frac{1}{9} & \frac{1}{9} & \frac{1}{9} & \frac{1}{9} & \frac{1}{36} & \frac{1}{36} & \frac{1}{36} & \frac{1}{36}
\end{array}\right]^{T} 1 \leqslant \alpha \leqslant 9 .
$$

The macroscopic quantities, namely, the density and momentum of each species, are obtained by computing the different moments of the distribution functions,

$$
\rho_{m}=\sum_{\alpha} f_{\alpha}^{m}, \quad \rho_{m} \mathbf{u}_{m}=\sum_{\alpha} f_{\alpha}^{m} \mathbf{e}_{\alpha}+\frac{\delta_{t}}{2} \mathcal{F}_{m} .
$$

The resulting macroscopic equations are the conservation equations for low Mach number flows subjected to a body force (for example, the gravity). In order to take into account the interaction of miscible species, we introduce diffusion forces. These forces are derived from kinetic theory by Kerkhof and Geboers [21],

$$
\mathcal{F}_{D, m}=-p \sum_{n=1}^{N} \frac{x_{m} x_{n}}{\mathcal{D}_{m n}}\left(\mathbf{u}_{m}-\mathbf{u}_{n}\right),
$$

and $\mathcal{F}_{m}$ becomes

$$
\mathcal{F}_{m}=\mathcal{F}_{D, m}+\mathcal{F}_{B, m}
$$

where $\mathcal{F}_{B, m}$ is a body force. As a result, the discrete kinetic equations (1) for the various species are coupled through $\mathcal{F}_{m}$. Since the diffusion force $\mathcal{F}_{D, m}$ depends on the velocity, total pressure $p$, molar fractions $x_{m}$, and (Maxwell-Stefan) diffusion coefficients $\mathcal{D}_{m n}$, a linear system must be solved at 
each time step in order to compute the species momentum by means of Eq. (6).

This force also called intermolecular friction force depends on the relative velocity of species. Thus, when all species have the same velocity, no diffusion occurs. The attempt to include the diffusion effects as a force acting on particles dates back to the early work on kinetic theory by Maxwell [27]. The same expression was rigorously derived later [20,28], and Kerkhof and Geboers present a more recent derivation in Ref. [21].

\section{MACROSCOPIC LIMIT}

\section{A. Macroscopic equations}

In this section we present the macroscopic limit of the proposed model via the Chapman-Enskog analysis. This multiple-scale expansion provides a relation between the mesoscopic scale of the Boltzmann equation and the macroscopic scale of the Navier-Stokes equation. This derivation is straightforward and is similar to the standard LBM with a force arising from Guo's forcing scheme (see Ref. [26]). Therefore in the low-Mach and continuum limit, the kinetic equation (1) and its moments (6) are equivalent to the following macroscopic equations:

$$
\begin{aligned}
& \partial_{t} \rho_{m}+\nabla \cdot\left(\rho_{m} \mathbf{u}_{m}\right)=0 \\
& \partial_{t}\left(\rho_{m} \mathbf{u}_{m}\right)+\nabla \cdot\left(\rho_{m} \mathbf{u}_{m} \otimes \mathbf{u}_{m}\right) \\
& =-\nabla p_{m}+\nabla \cdot\left\{\mu_{m}\left[\nabla \mathbf{u}_{m}+\left(\nabla \mathbf{u}_{m}\right)^{T}\right]\right\} \\
& \quad-p \sum_{n=1}^{N} \frac{x_{m} x_{n}}{\mathcal{D}_{m n}}\left(\mathbf{u}_{m}-\mathbf{u}_{n}\right)+\mathcal{F}_{B, m},
\end{aligned}
$$

where the partial pressure is equal to $p_{m}=\rho_{m} c_{s}^{2}$, and the total pressure is given by Dalton's law $p=\sum_{m=1}^{N} p_{m}$. The dynamic viscosity of species $m$ is expressed in terms of the relaxation time according to

$$
\mu_{m}=\rho_{m} c_{s}^{2}\left(\tau_{m}-\frac{\delta_{t}}{2}\right) .
$$

\section{B. Limit expression}

We now focus on the limit expressions of the previous equations. Using the dimensionless formulations of Eqs. (9) and (10), one can estimate, a priori, the order of magnitude of each term. In the following, we will show that the MaxwellStefan equations are recovered in the case of pure diffusion. We first begin with the intermolecular friction force, which yields to the estimate

$$
\mathcal{F}_{D, m}=-p \sum_{n=1}^{N} \frac{x_{m} x_{n}}{\mathcal{D}_{m n}}\left(\mathbf{u}_{m}-\mathbf{u}_{n}\right)=O\left(p_{\text {ref }} u_{\Delta} / \mathcal{D}_{\text {ref }}\right),
$$

where $u_{\Delta}$ is an appropriate velocity difference between species, and quantities with the ref subscript represent an estimate of their order of magnitude. In a similar way, we have

$$
\begin{aligned}
\partial_{t}\left(\rho_{m} \mathbf{u}_{m}\right) & =O\left(\rho_{\mathrm{ref}} u_{\mathrm{ref}} / t_{\mathrm{ref}}\right), \\
\nabla \cdot\left(\rho_{m} \mathbf{u}_{m} \otimes \mathbf{u}_{m}\right) & =O\left(\rho_{\mathrm{ref}} u_{\mathrm{ref}}^{2} / L_{\mathrm{ref}}\right), \\
\nabla p_{m} & =O\left(p_{\mathrm{ref}} / L_{\mathrm{ref}}\right), \\
\nabla \cdot\left\{\mu_{m}\left[\nabla \mathbf{u}_{m}+\left(\nabla \mathbf{u}_{m}\right)^{T}\right]\right\} & =O\left(\mu_{\mathrm{ref}} u_{\mathrm{ref}} / L_{\mathrm{ref}}^{2}\right) .
\end{aligned}
$$

The species momentum convection can be neglected compared to the diffusion if Eq. (14) $\ll$ Eq. (12), i.e., $\mathrm{Ma}^{2} \ll \mathrm{Pe}_{\Delta}$, where the Mach number is defined as $\mathrm{Ma}=u_{\text {ref }} / c_{s, \text { ref }}$ and the Péclet number is $\mathrm{Pe}=L_{\mathrm{ref}} u_{\Delta} / \mathcal{D}_{\text {ref }}$. Similarly, the shear forces are negligible if Eq. (16) $\ll$ Eq. (12), i.e., $\mathrm{Ma}^{2} \ll \mathrm{Pe}_{\Delta}$ Re with $\operatorname{Re}=L_{\text {ref }} u_{\text {ref }} / v_{\text {ref }}$. As $u_{\Delta}$ may change during the mixing, the influence of the different terms in Eq. (10) may vary in time and space.

Let assume a low Mach flow whose dynamic is mostly diffusive. We can neglect the contributions associated with convection $\left(\mathrm{Ma}^{2} \ll \mathrm{Pe}_{\Delta}\right)$ and shear forces $\left(\mathrm{Ma}^{2} \ll \mathrm{Pe}_{\Delta} \mathrm{Re}\right)$. In addition, the characteristic time is given by $t_{\text {ref }}=L_{\text {ref }}^{2} / \mathcal{D}_{\text {ref }}$ and $u_{\text {ref }}=u_{\Delta}$, and thereby the species acceleration can also be neglected, Eq. (13) « Eq. (12). Hence, Eq. (10) reduces to

$$
\nabla p_{m}=p \sum_{n=1}^{N} \frac{x_{m} x_{n}}{\mathcal{D}_{m n}}\left(\mathbf{u}_{n}-\mathbf{u}_{m}\right)
$$

If we sum this equation over all species, we obtain

$$
\nabla p=0 .
$$

As a result for this specific case, the process is isobaric. Using Dalton's law, Maxwell-Stefan's equations are easily recovered from Eq. (17):

$$
\nabla x_{m}=\sum_{n=1}^{N} \frac{x_{m} x_{n}}{\mathcal{D}_{m n}}\left(\mathbf{u}_{n}-\mathbf{u}_{m}\right)=\sum_{n=1}^{N} \frac{x_{m} \mathbf{N}_{n}-x_{n} \mathbf{N}_{m}}{c_{t} \mathcal{D}_{m n}},
$$

where we have introduced the species molar concentration $c_{m}=x_{m} c_{t}$ and the species molar flux $\mathbf{N}_{m}=c_{m} \mathbf{u}_{m}, c_{t}$ being the mixture molar concentration. In the case of an equimolar binary mixture, Fick's law is obtained, $\mathbf{N}_{1}=-c_{t} \mathcal{D}_{12} \nabla x_{1}$ or $\mathbf{u}_{1}=-x_{1}^{-1} \mathcal{D}_{12} \nabla x_{1}$.

\section{Transport coefficients}

In the previous subsections, the equivalent macroscopic equations were presented. However, the transport coefficients, viscosities, and diffusion coefficients have still to be defined.

The lattice Boltzmann scheme still retains a connection with the macroscopic scale through the relation between the relaxation time and the viscosity stemming from the Chapman-Enskog expansion [Eq. (11)]. Since each species has its own kinetic equation [Eq. (1)], $N$ relaxation times (i.e., viscosities) need to be defined, and a relation between the mixture properties and species viscosity has to be specified. Some of the previous lattice Boltzmann multicomponent models disregard this issue ([4,7]), and others set the viscosities of each species equal to the mixture viscosity $([8,9,14,16,18])$ or use Wilke's law ([12,13]).

In the following, we present the submodel for the transport coefficients that we derive in the framework of the multifluid approach that constitutes one of the main features of our model. Expression for the transport coefficients for a dilute gas can be obtained by kinetic theory [20]. By extending the works of Hirschfelder, Curtiss, and Bird to mixtures, Kerkhof and Geboers [21] define diffusion coefficients and species partial viscosities in terms of the molecular properties, temperature, and composition of the mixture.

In order to avoid confusion between pure viscosity and the viscosity of a species $m$ in the mixture, we refer to the 
latter as the partial viscosity. Following Kerkhof and Geboers, the partial viscosities can be computed by solving the linear system

$$
\left(\mu_{m}\right)=\left[P_{m n}\right]^{-1}\left(\begin{array}{c}
1 \\
\vdots \\
1
\end{array}\right)
$$

with

$$
\begin{aligned}
P_{m m}= & \frac{2}{k_{B} T}\left[\frac{4}{5} \Omega_{m m}^{(2,2)}+\sum_{n \neq m}^{N} \frac{x_{n}}{x_{m}} \frac{16}{15} \frac{\mathcal{M}_{n}}{\left(\mathcal{M}_{m}+\mathcal{M}_{n}\right)^{2}}\right. \\
& \left.\times\left(5 \mathcal{M}_{m} \Omega_{m n}^{(1,1)}+\frac{3}{2} \mathcal{M}_{n} \Omega_{m n}^{(2,2)}\right)\right]
\end{aligned}
$$

and for off-diagonal elements $(n \neq m)$,

$$
P_{m n}=-\frac{2}{k_{B} T}\left[\frac{16}{15} \frac{\mathcal{M}_{m} \mathcal{M}_{n}}{\left(\mathcal{M}_{m}+\mathcal{M}_{n}\right)^{2}}\left(5 \Omega_{m n}^{(1,1)}-\frac{3}{2} \Omega_{m n}^{(2,2)}\right)\right],
$$

where $k_{B}$ is the Boltzmann constant, $T$ the temperature, $\mathcal{M}_{m}$ the mass of a single molecule for the species $m, x_{m}$ the mole fraction, and the $\Omega$ integrals as defined in Ref. [20] and depend on the temperature and the molecular properties based on the Lennard-Jones potential (see the Appendix for details).

Following the same assumptions made in Ref. [20] (Sec. 8.2.iii) and in Ref. [29], the previous linear system can be simplified. Thereby, the partial viscosities depend on the composition of the mixture and can be expressed in terms of the molar fractions, the pure viscosities $\mu_{0, m}$, and the species molar masses, and Wilke's formula is recovered yielding to

$$
\mu_{m}=\frac{x_{m} \mu_{0, m}}{\sum_{n}^{N} x_{n} \Phi_{m n}}
$$

with

$$
\Phi_{m n}=\frac{1}{2 \sqrt{2}}\left(1+\frac{M_{m}}{M_{n}}\right)^{-\frac{1}{2}}\left[1+\left(\frac{\mu_{0, m}}{\mu_{0, n}}\right)^{\frac{1}{2}}\left(\frac{M_{n}}{M_{m}}\right)^{\frac{1}{4}}\right]^{2},
$$

where $\mathcal{M}_{m}=M_{m} / \mathcal{N}_{a}$, with $\mathcal{N}_{a}$ the Avogadro number and $M_{m}$ the molar mass of species $m$. We point out that in kinetic theory, the pure viscosity has the following expression:

$$
\mu_{0, m}=\frac{5 k_{B} T}{8 \Omega_{m m}^{(2,2)}},
$$

which is asymptotically consistent with Eqs. (21) and (22).

For the Maxwell-Stefan diffusion coefficients, we use the same expression obtained from the classical kinetic theory of gases [20]:

$$
\mathcal{D}_{m n}=\mathcal{D}_{n m}=\frac{3\left(\mathcal{M}_{m}+\mathcal{M}_{n}\right)}{16 p \mathcal{M}_{m} \mathcal{M}_{n}} \frac{\left(k_{B} T\right)^{2}}{\Omega_{m n}^{(1,1)}} .
$$

The diffusion coefficients are usually taken as constant at a given reference pressure and temperature since the pressure variation is not significant. In practice, the transport coefficients, pure viscosities, and diffusion coefficients can be set according to different strategies. For instance, they can be directly chosen in lattice units (as in Secs. V A and V B) depending on a given dimensionless number (Reynolds number, Péclet number, etc). In some cases, experimental values are available (as in Sec. V C), or else the previous equations can be employed (as in Sec. V D).

\section{SPECIES WITH DIFFERENT MOLECULAR MASSES}

In the standard lattice Boltzmann equation, the pseudo(isothermal) speed of sound $\left(c_{s}\right)$ is fixed by the lattice. For the D2Q9 velocity set, $c_{s}$ is equal to $c_{s}^{2}=1 / 3$ (in lattice units for a reference temperature and molecular mass) for all species, which is not the case for mixture of species having different molecular masses. Indeed, recalling that the partial pressure obeys the ideal gas law, from the definition of the isothermal speed of sound of a species $\left[c_{s, m}^{2}=\left(\frac{\partial p_{m}}{\partial \rho_{m}}\right)_{T}\right]$, one finds $c_{s, m}^{2}=R T / M_{m}$ and $p_{m}=\rho_{m} c_{s, m}^{2}$, where $R$ is the universal gas constant.

In order to account for the differences in the species pseudospeed of sound, one can modify the equilibrium distribution functions [30]. However, as shown by those authors, the maximum molecular mass ratio is limited to three. Furthermore, this approach adds errors in the viscous stress tensor, which can be reduced by increasing the velocity set from D2Q9 to D2Q13 [31]. Nonetheless, the molar mass ratio is still limited to three because of stability issues, and expanding the number of velocities makes the algorithm more complex and costly. Another strategy is to set the pseudospeed of sound in terms of the minimum molecular mass [30]. Thus, in one time step, the lightest species streams exactly to the next lattice point, while the heavier species stream between the original lattice point and the next one. Populations for the heavier species are then interpolated to the next lattice point. This process can simulate binary diffusion with molar mass ratio up to nine before the accuracy decreases because of the interpolation that adds numerical diffusion. This approach is very expensive. Indeed, it requires the use of an interpolation scheme for each species populations in each kinetic velocity directions (i.e., eight in D2Q9).

In the present study, a variable pseudospeed of sound is introduced through a body force [32]. This strategy is simple since neither interpolation, nor extended velocity set, nor modified equilibrium is required. In particular, Guo's forcing scheme [26] is used, and the forcing term is calculated according to

$$
\mathcal{F}_{B, m}=\left(1-\beta_{m}\right) c_{s}^{2} \nabla \rho_{m},
$$

where the gradient term is computed by means of a compact scheme

$$
\nabla \rho_{m}(\mathbf{x})=\frac{1}{c_{s}^{2}} \sum_{\alpha} \omega_{\alpha} \mathbf{e}_{\alpha} \rho_{m}\left(\mathbf{x}+\mathbf{e}_{\alpha}\right) .
$$

The partial pressure then becomes

$$
p_{m}=\beta_{m} c_{s}^{2} \rho_{m},
$$

where $\beta_{m}=c_{s, m}^{2} / c_{s}^{2}$ is the ratio between the species and the standard LBM pseudospeed of sound. This forcing strategy changes only the equation of state, and the relation between the partial pressure and density is modified according to the 

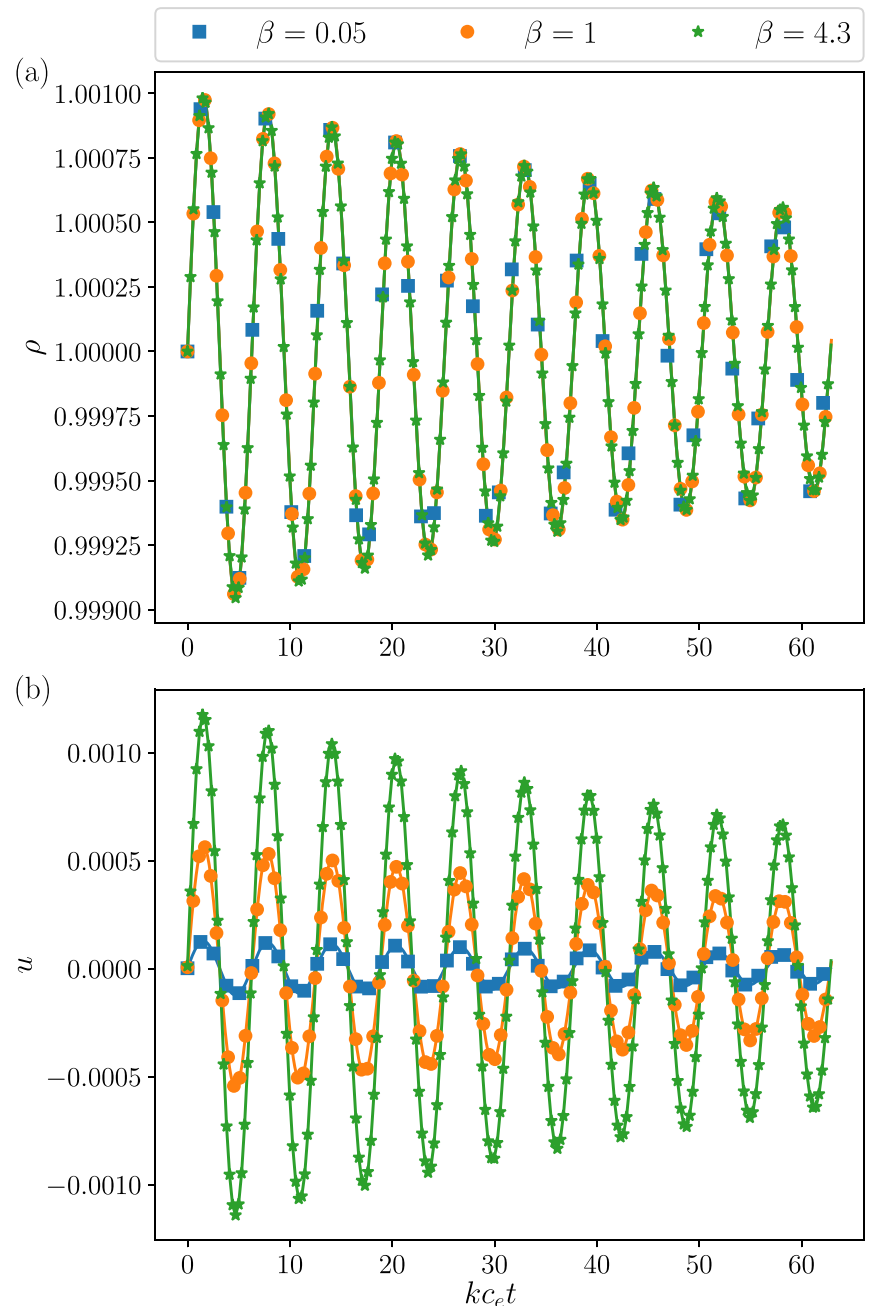

FIG. 1. (a) Temporal evolution of $\rho$ and $u$ (b) at $n_{x} / 4$ for $\beta=$ $0.05,1,4.3$ and $n_{x}=128$. Symbols and lines stand for simulation results and analytical solutions from Eqs. (30) and (31), respectively.

molecular mass of the species. This approach has also the advantage that the partial viscosity [Eq. (11)] is always defined in terms of the standard LBM pseudospeed of sound $c_{s}$. In practice, we usually define a reference species $n$ whose speed of sound is the same as $c_{s}$ equal to $1 / 3$ in lattice units $\left(\beta_{n}=1\right.$, $\left.p_{n}=\rho_{n} c_{s}^{2}=\rho_{n} R T / M_{n}\right)$, and $\beta_{m}=M_{n} / M_{m}$ are then the ratio of molecular masses $\left(p_{m}=\rho_{m} c_{s, m}^{2}=\rho_{m} c_{s}^{2} \beta_{m}=\rho_{m} R T / M_{m}\right)$.

\section{NUMERICAL SIMULATIONS}

In order to validate the proposed model, we present four two-dimensional cases, referred to as A, B, C, and D. In case A, the forcing-term approach is applied to the free decay of a density wave. In case B, two species having the same molecular mass diffuse in each other. These two cases are selected to assess the numerical capabilities of the proposed method, and the results are validated against analytical results. Then Loschmidt's tube experiment is reproduced in case C, which corresponds to the diffusion of a ternary mixture with different molecular masses. In case D we simulate the interaction of two multicomponent opposed jets. In all cases the simulation is initialized with the equilibrium distribution (2).
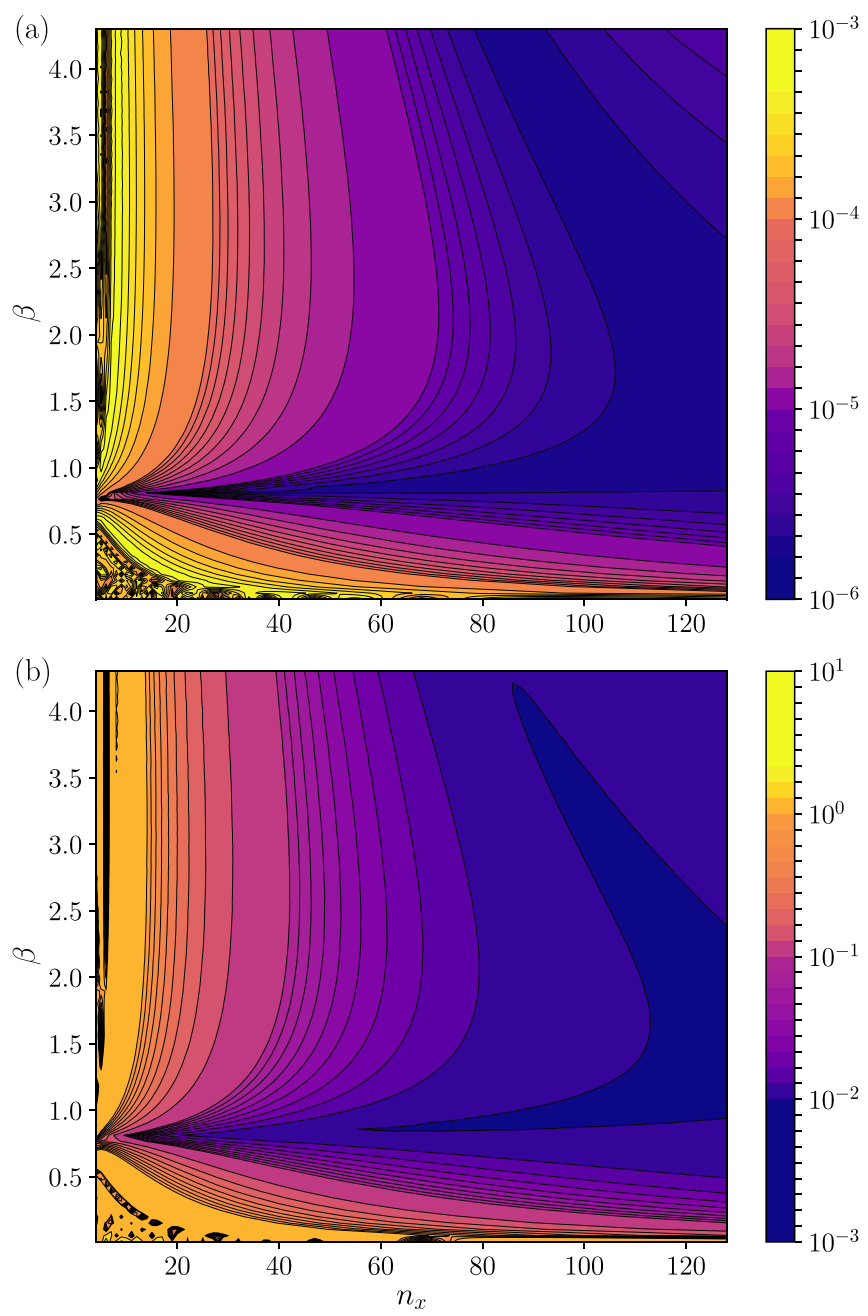

FIG. 2. (a) Relative errors of the density $\varepsilon_{\rho}$ and velocity $\varepsilon_{u}$ (b).

\section{A. Decay of a density wave}

The accuracy of the forcing strategy to define the species pseudospeed of sound is assessed by simulating a singlespecies flow corresponding to the decay of a free density wave damped by a low viscosity as proposed in Ref. [31]. By considering small perturbations of density and velocity from the linearized Navier-Stokes equations, the density and the velocity of the damped wave are given by

$$
\begin{aligned}
& \rho=\rho_{0}+\delta_{\rho} \exp \left(w_{i} t\right) \cos \left(k x-w_{r} t\right) \\
& u=\frac{\delta_{\rho}}{k} \exp \left(w_{i} t\right)\left[w_{r} \cos \left(k x-w_{r} t\right)-w_{i} \sin \left(k x-w_{r} t\right)\right]
\end{aligned}
$$

We perform simulations assuming a two-dimensional periodic domain $\left(n_{x}, n_{y}\right)$. In order to compare our results with the analytical solution, we introduce the equivalent species pseudospeed of sound $c_{e}=\sqrt{\beta} c_{s}$ and $v$ the kinematic viscosity of the fluid, and set $\rho_{0}=1$ (average density), $\delta_{\rho}=10^{-3}$ (amplitude of the density perturbation wave), and $\nu k / c_{e}=10^{-2}, k$ being the wave number $k=2 \pi / n_{x}$. In the harmonic decomposition, the dispersion relation yields a wave frequency that 


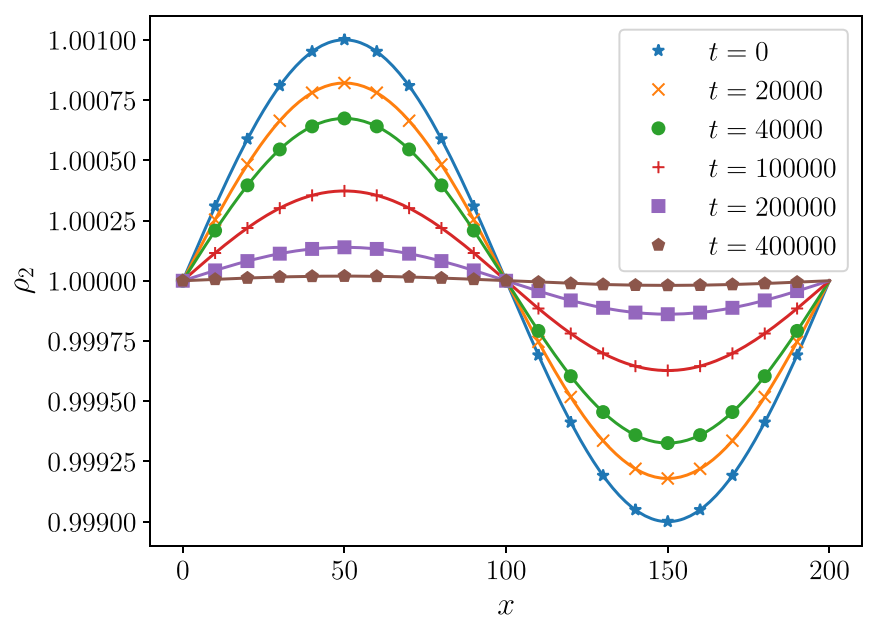

FIG. 3. Evolution of $\rho_{2}$ for $\tau=1$ and $\mathcal{D}=10^{-2}$ at different iterations. Symbols and lines stand for simulation results and analytical solutions from Eq. (37), respectively.

is split into a real and an imaginary part:

$$
\begin{aligned}
w & =w_{r}+i w_{i}, \\
w_{r} & = \pm k c_{e} \sqrt{1-v^{2} k^{2} / c_{e}^{2}}, \\
w_{i} & =-k c_{e}\left(v k / c_{e}\right) .
\end{aligned}
$$

Simulations are carried out for different domain sizes $n_{x}$ at various speed of sound ratios $\beta$ while keeping $n_{y}=5$ constant. As an example, we plot in Fig. 1 the temporal evolution of $\rho$ and $u$ at $n_{x} / 4$ for $\beta=0.05,1,4.3$ and $n_{x}=128$. The dissipation error is undetectable, and a small dispersion error is visible only for $\beta=0.05$.

The relative global error of the density and the velocity field is defined in terms of the L2 norm,

$$
\varepsilon_{\phi}=\sqrt{\frac{\sum_{i}^{n_{x}}\left(\phi_{i}-\phi_{\text {analytical }, i}\right)^{2}}{\sum_{i}^{n_{x}} \phi_{\text {analytical }, i}^{2}}},
$$

$\phi$ standing for either density or velocity. The results are compared at nondimensional time $k t c_{e}=10 \times 2 \pi$ and reported in Fig. 2 for various $\beta$ and $n_{x}$. The model is found to be stable

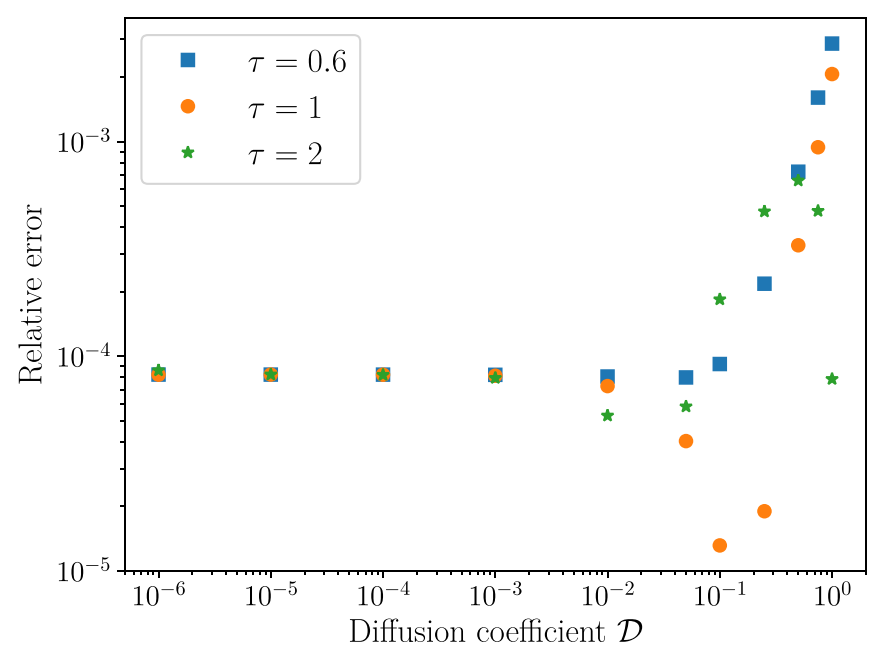

FIG. 4. Relative errors of the diffusion coefficient.
TABLE I. Initial molar fractions; in our simulation we take $\delta=$ $5 \times 10^{-4}$ (in the experiment, $\delta=0$ ).

\begin{tabular}{lcccc}
\hline \hline & Tube & $x_{\mathrm{Ar}}$ & $x_{\mathrm{CH}_{4}}$ & $x_{\mathrm{H}_{2}}$ \\
\hline Left & $\left(0<x<L_{\text {ref }} / 2\right)$ & $0.509-\delta$ & $2 \delta$ & $0.491-\delta$ \\
Right & $\left(L_{\text {ref }} / 2<x<L_{\text {ref }}\right)$ & $0.485-\delta$ & $0.515-\delta$ & $2 \delta$ \\
\hline \hline
\end{tabular}

in the range $0.05 \leqslant \beta \leqslant 4.3$, indicating that our model is able to simulate molar mass ratios up to 86 with small errors on the density and velocity depending on the size of the grid and on the value of $\beta$. With the present strategy, the relative errors are lower, and the range of stable $\beta$ values is greater in comparison to the modifications of the equilibrium and the use of a larger velocity set as proposed in Ref. [31]. In addition, the present approach is also easier to implement compared to the interpolation of distribution functions, which can be cumbersome to code in three dimensions, especially on the boundary nodes.

\section{B. Equimolar counterdiffusion}

In this test case, we study the mixing between two species of equal molecular masses for which the mass transfer occurs only by diffusion, and governing equations are

$$
\partial_{t} \rho_{m}=\mathcal{D} \nabla^{2} \rho_{m}, \quad \text { for } \quad m=1,2 .
$$

A particular solution of Eq. (36) is

$$
\rho_{m}(x, t)=\rho_{0}+(-1)^{m} \delta_{\rho} \exp \left(-k^{2} \mathcal{D} t\right) \sin (k x) .
$$

We choose $\rho_{0}=1, \delta_{\rho}=10^{-3}, k=2 \pi / n_{x}$, and use a twodimensional periodic domain $\left(n_{x}=200, n_{y}=5\right)$. As an example, we plot in Fig. 3 the temporal evolution of $\rho_{2}$ for $\mathcal{D}=10^{-2}$ and $\tau=1$ for both species. Numerical results are indistinguishable from the analytical solution (37).

For comparison, we have evaluated the error of the diffusion coefficient at various $\mathcal{D}$ and relaxation times $\tau$ (which is assumed to be the same for the two species). The relative error $\left\|\mathcal{D}_{\text {num }}-\mathcal{D}\right\| / \mathcal{D}$ is reported in Fig. 4 where $\mathcal{D}_{\text {num }}$ is computed at $x=n_{x} / 4$ by linear fit of Eq. (37) and $\mathcal{D}$ is set using Eq. (7). The numerical solution is in good agreement with the theoretical results for $\mathcal{D}<0.1$ and for all relaxation

\begin{tabular}{|c|c|c|c|c|}
\hline \multirow[t]{2}{*}{$\begin{array}{l}L_{\mathrm{ref}} \\
p \\
T \\
T\end{array}$} & \multirow[t]{2}{*}{$\begin{array}{l}{[\mathrm{m}]} \\
{[\mathrm{Pa}]} \\
{[\mathrm{K}]}\end{array}$} & \multicolumn{3}{|c|}{$\begin{array}{c}2 \sqrt{1 / 60} \pi \\
101325 \\
307.15\end{array}$} \\
\hline & & $\mathrm{Ar}$ & $\mathrm{CH}_{4}$ & $\mathrm{H}_{2}$ \\
\hline$M_{m}$ & {$[\mathrm{~g} / \mathrm{mol}]$} & 39.948 & 16.0425 & 2.01588 \\
\hline $\mathcal{D}_{\mathrm{Ar} m}$ & {$\left[\mathrm{~mm}^{2} / \mathrm{s}\right]$} & - & 21.57 & 83.35 \\
\hline $\mathcal{D}_{\mathrm{CH}_{4} m}$ & {$\left[\mathrm{~mm}^{2} / \mathrm{s}\right]$} & 21.57 & - & 77.16 \\
\hline $\mathcal{D}_{\mathrm{H}_{2} m}$ & {$\left[\mathrm{~mm}^{2} / \mathrm{s}\right]$} & 83.35 & 77.16 & - \\
\hline$\mu_{0, m}$ & {$[\mu \mathrm{Pa} / \mathrm{s}]$} & 22.83 & 11.35 & 9.18 \\
\hline
\end{tabular}
times. We note that the relative error in density, $\varepsilon_{\rho}$ as defined in Eq. (35), is three orders of magnitude smaller than the relative errors of the diffusion coefficient. The figure shows a discrepancy between the numerical and theoretical results

TABLE II. Physical parameters of the experiment. 


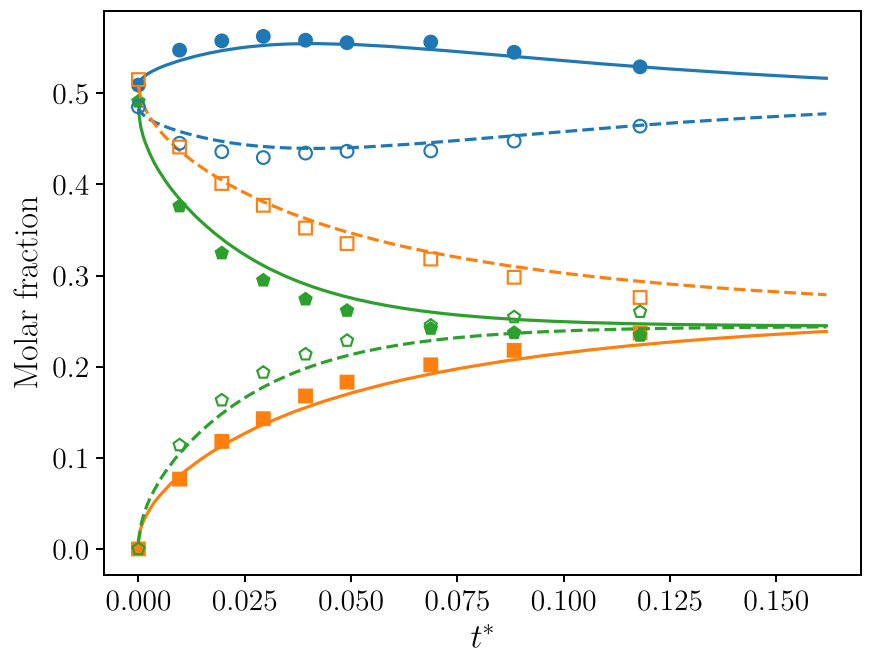

FIG. 5. Comparison between simulation (lines) and experimental data (symbols) extracted from Ref. [36]. Molar fraction of argon, blue; molar fraction of methane, orange; molar fraction of hydrogen, green. Solid lines and filled symbols, left tube; dashed lines and unfilled symbols, right tube.

for $\mathcal{D}>0.1$. Having kept constant the size of the domain for all $\mathcal{D}$, this discrepancy is likely to be ascribed to a loss of spatial and temporal resolution. Nonetheless the relative error of the diffusion coefficient is always less than $0.4 \%$ for all $\tau$. Different sizes of domain are used (not shown in the present study), and we recover the classic second-order accuracy in space indicating that the forcing approach does not deteriorate the accuracy of the LBM algorithm.

\section{Loschmidt's tube}

Having validated the ability of the method to simulate the decay of a single-species density wave (at various different

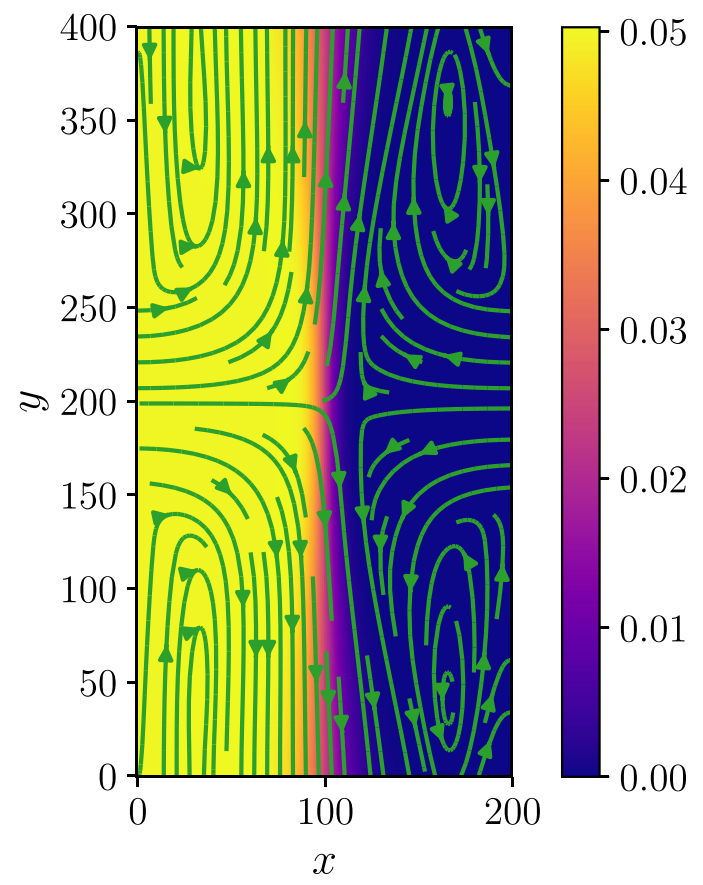

FIG. 6. Molar fraction and velocity streamline plot of $\mathrm{H}_{2} \mathrm{O}$.
TABLE III. Initial molar fractions; in our simulation we take $\delta=$ $10^{-5}$ (in the experiment, $\delta=0$ ).

\begin{tabular}{lcccc}
\hline \hline Stream & $x_{\mathrm{H}_{2}}$ & $x_{\mathrm{N}_{2}}$ & $x_{\mathrm{O}_{2}}$ & $x_{\mathrm{H}_{2} \mathrm{O}}$ \\
\hline Left & 0.10 & $0.85-\delta$ & $0+\delta$ & 0.05 \\
Right & $0+\delta$ & $0.9-2 \delta$ & 0.10 & $0+\delta$ \\
\hline \hline
\end{tabular}

molar masses), and the counterdiffusion of species having equal molecular masses (at various diffusion coefficients), we simulate the Loschmidt's tube experiment [33]. The latter consists of the mixing of a ternary mixture of gases having different molecular masses (argon, methane, and hydrogen). For such a process, a diffusion reversal is observed depending on the initial species composition [34]. In particular, we have considered two tubes of the same dimension filled with mixtures of different composition in the left and right tubes that are joined at the beginning of the experiment. In the experiment the left and right mean composition is measured in time during the mixing. In the experimental apparatus, the length of each tube is $L_{\mathrm{ref}} / 2 \approx 0.405$, and the period of observation is approximately $1 \mathrm{~h}$. The initial molar fractions are given in Table I, and the other physical parameters are summarized in Table II.

The simulation is carried out on a domain size of $\left(n_{x}, n_{y}\right)=$ $(200,25)$. Classical bounce-back rules are used on the left and right boundaries, and periodic conditions are applied on the top and bottom sides of the domain.

For computational purposes, we have rescaled the Maxwell-Stefan diffusion coefficients by a factor $10^{3}$, and the partial viscosities have been computed imposing the same species Schmidt numbers, which we define in terms of the pure viscosity as in the experiment.

Figure 5 reports the evolution of the mean molar fractions for the left and right tubes in nondimensional time units $t^{*}=$ $t \times \mathcal{D}_{\mathrm{ArCH}_{4}} /\left(L_{\mathrm{ref}}\right)^{2}$. The mean molar fractions are in very good agreement with the experimental data. As expected, initially argon diffuses in the same direction of the concentration gradient, contrary to the behavior predicted by using Fick's law. This reverse diffusion occurs on a scale of approximately 0.04 nondimensional time units, and the concentration of argon attains a plateau in both tubes in spite of the presence of large concentration gradients. The other species do not exhibit such a diffusion barrier. At later times, Fick's-like diffusion takes place. It is important to point out that this complex diffusion dynamics can be recovered only by the Maxwell-Stephan equations [35].

\section{Opposed jets flow}

The model is finally validated for a flow whose dynamics is dominated by a convection-diffusion competing mechanism.

TABLE IV. Kinetic constants from Ref. [20].

\begin{tabular}{lccccc}
\hline \hline & $m$ & $\mathrm{H}_{2}$ & $\mathrm{~N}_{2}$ & $\mathrm{O}_{2}$ & $\mathrm{H}_{2} \mathrm{O}$ \\
\hline$M_{m}$ & {$[\mathrm{~g} / \mathrm{mol}]$} & 2.01588 & 28.0134 & 31.9988 & 18.0153 \\
$\epsilon_{m} / k_{B}$ & {$[\mathrm{~K}]$} & 36.7 & 47.6 & 113 & 775 \\
$\sigma_{m}$ & {$[\mathrm{~nm}]$} & 0.2959 & 0.385 & 0.433 & 0.252 \\
\hline \hline
\end{tabular}




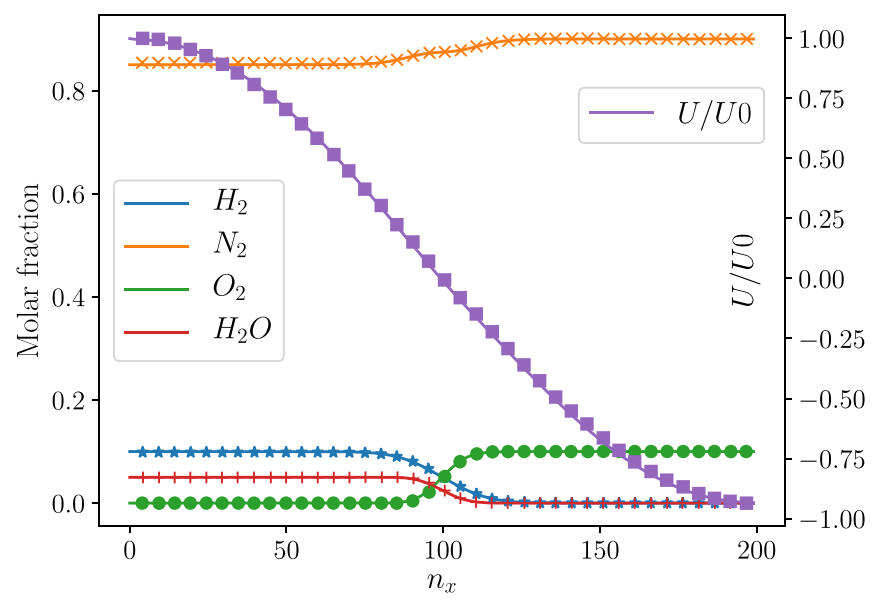

FIG. 7. Comparison between the present method (line) and LBM from Ref. [12] (symbols) at $y=n_{y} / 2$.

The test case that we investigate is the same proposed in Ref. [12], which consists of two opposed jets of quaternary mixtures of gases having different initial concentrations (see Fig. 6 and Table III). The domain size is $\left(n_{x}, n_{y}\right)=(200,400)$ and the widths of the left and right incoming streams are the same and equal to $0.4 n_{y}$. At left and right boundaries, equilibrium is assumed for the distribution functions, and the velocity is set equal to zero. For the left and right incoming streams, we assume that all species velocities are respectively equal to $U_{L}=U_{0}$ and $U_{R}=-0.936 U_{0}$ (as inferred from Fig. 3 of Ref. [12]), and we chose $U_{0}=0.04$. At the top and bottom boundaries, the outer incoming distribution functions are extrapolated from the interior. The transport coefficients are evaluated by means of Eqs. (23)-(26) at atmospheric pressure and temperature $T=300 \mathrm{~K}$. The species kinetic constants needed to evaluate the $\Omega$ integrals are given in Table IV.

Figure 7 shows the distributions of the molar fractions and the mixture mass velocity at the symmetry plane $\left(y=n_{y} / 2\right)$ and at steady state. The results are in close agreement with Ref. [12] where the CHEMKIN package is used to calculate the transport coefficients and mixture-averaged diffusion coefficients are employed for each species. Despite using a simplifying mixture-average diffusion, the results are similar since the multicomponent diffusion effects, which cannot be captured with this assumption, are not significant. In addition, these transitory complex diffusion phenomena as in the case of the Loschmidt's tube experiment (Sec. VC) would not be visible on this steady-state comparison. Figure 7 confirms that our model correctly predicts the dynamics of the flow characterized by a competing convection-diffusion mechanism.

\section{CONCLUSION}

In the present paper, we derive, explain, and validate a LBM for miscible gases. We show that the mixing dynamics of multispecies mixtures can be simulated by a forcing term in the lattice Boltzmann algorithm [Eq. (7)] and the addition of a body force to account for species having different molecular masses [Eq. (27)]. Furthermore, the model also relies on the use of transport coefficients that are calculated by an approximation of the relations obtained from kinetic theory [Eq. (23)].

The model is validated against analytical, experimental, and numerical results available in the literature. We have shown that the model can accurately simulate the decay of a density wave for a variety of pseudospeed of sound corresponding to molar mass ratios up to 86 . The model adequately predicts the diffusion process in binary and ternary mixtures of gases as shown for the case of the equimolar counterdiffusion and Loschmidt's tube experiment. Complex diffusion phenomena such as reverse diffusion occur in ternary mixtures. These phenomena are well observed in our model, and the dynamics predicted by the Maxwell-Stefan equations is correctly recovered. The present model also adequately predicts the dynamics of flows where convection and diffusion compete as in the case of two opposed jets of mixtures.

Finally, one of the advantages of the forcing approach is the easiness of implementation. Since collision is not altered, the method that we propose can easily be introduced in any other lattice Boltzmann algorithms in order to take into account complex diffusion among species. Upcoming applications of the present model will focus on instabilities resulting from the transient mixing dynamics in porous media.

\section{APPENDIX: $\Omega$ INTEGRALS}

We specify the expression of the $\Omega$ integrals. We recall that the interactions of a similar pair of molecules can be approximated by the Lennard-Jones potential,

$$
\varphi(r)=4 \epsilon\left[\left(\frac{\sigma}{r}\right)^{12}-\left(\frac{\sigma}{r}\right)^{6}\right]
$$

where $r$ is the distance between the molecules, $\sigma$ the collision diameter, and $\epsilon$ the depth of the potential well. For a dissimilar $(m, n)$ pair of molecules, we use the following standard mixing rules:

$$
\begin{aligned}
\sigma_{m n} & =\left(\sigma_{m}+\sigma_{n}\right) / 2, \\
\epsilon_{m n} & =\sqrt{\epsilon_{m} \epsilon_{n}}, \\
\frac{1}{\psi_{m n}} & =\frac{1}{\mathcal{M}_{m}}+\frac{1}{\mathcal{M}_{n}} .
\end{aligned}
$$

Unlike the rigid sphere model, the Lennard-Jones potential is a physically realistic potential, and the $\Omega$ integrals cannot be calculated analytically. However, we compute the $\Omega$ integrals for the rigid sphere and introduce the $\Omega^{\star}$ ratio, which embodies the deviation of the $\Omega$ integrals between the Lennard-Jones and rigid sphere potentials,

$$
\Omega_{m n}^{\star(i, j)}=\Omega_{m n}^{(i, j)} / \Omega_{m n}^{r s(i, j)},
$$

with

$$
\Omega_{m n}^{r s(i, j)}=\sqrt{\frac{k_{B} T}{2 \pi \psi_{m n}}} \frac{(j+1) !}{2}\left[1-\frac{1}{2} \frac{1+(-1)^{i}}{1+i}\right] \pi\left(\sigma_{m n}\right)^{2} .
$$


$\Omega_{m n}^{\star(i, j)}$ depends only on $T^{\star}=T k_{B} / \epsilon_{m n}$, and its value is obtained from a fitted curve computed from a numerical integration [21]. For more details about the potentials, we invite the reader to read Chap. 8 of Ref. [20].
Despite that the derivation is rigorously correct only for dilute gases, we could to a certain extent apply these results to liquids and dense gases by means of the Eyring and Enskog theories, respectively.
[1] S. A. Hosseini, N. Darabiha, and D. Thévenin, Mass-conserving advection-diffusion lattice Boltzmann model for multi-species reacting flows, Phys. A 499, 40 (2018).

[2] T. Krüger, H. Kusumaatmaja, A. Kuzmin, O. Shardt, G. Silva, and E. M. Viggen, The Lattice Boltzmann Method, Principles and Practice (Springer, Cham, 2017).

[3] P. L. Bhatnagar, E. P. Gross, and M. Krook, A model for collision processes in gases. I. Small amplitude processes in charged and neutral one-component systems, Phys. Rev. 94, 511 (1954).

[4] L.-S. Luo and S. S. Girimaji, Theory of the lattice Boltzmann method: Two-fluid model for binary mixtures, Phys. Rev. E 67, 036302 (2003).

[5] L. Sirovich, Kinetic modeling of gas mixtures, Phys. Fluids 5, 908 (1962).

[6] A. S. Joshi, A. A. Peracchio, K. N. Grew, and W. K. S. Chiu, Lattice Boltzmann method for continuum, multi-component mass diffusion in complex 2D geometries, J. Phys. D 40, 2961 (2007).

[7] Z.-X. Tong, Y.-L. He, L. Chen, and T. Xie, A multi-component lattice Boltzmann method in consistent with Stefan-Maxwell equations: Derivation, validation and application in porous medium, Comput. Fluids 105, 155 (2014).

[8] P. Asinari, Semi-implicit-linearized multiple-relaxation-time formulation of lattice Boltzmann schemes for mixture modeling, Phys. Rev. E 73, 056705 (2006).

[9] P. Asinari and L.-S. Luo, A consistent lattice Boltzmann equation with baroclinic coupling for mixtures, J. Comput. Phys. 227, 3878 (2008).

[10] B. B. Hamel, Kinetic model for binary gas mixtures, Phys. Fluids 8, 418 (1965).

[11] V. Sofonea and R. F. Sekerka, BGK models for diffusion in isothermal binary fluid systems, Phys. A 299, 494 (2001).

[12] S. Arcidiacono, I. V. Karlin, J. Mantzaras, and C. E. Frouzakis, Lattice Boltzmann model for the simulation of multicomponent mixtures, Phys. Rev. E 76, 046703 (2007).

[13] J. Kang, N. I. Prasianakis, and J. Mantzaras, Thermal multicomponent lattice Boltzmann model for catalytic reactive flows, Phys. Rev. E 89, 063310 (2014).

[14] P. Asinari, Multiple-relaxation-time lattice Boltzmann scheme for homogeneous mixture flows with external force, Phys. Rev. E 77, 056706 (2008).

[15] P. Andries, K. Aoki, and B. Perthame, A consistent BGK-type model for gas mixtures, J. Stat. Phys. 106, 993 (2002).

[16] P. Asinari, Lattice Boltzmann scheme for mixture modeling: Analysis of the continuum diffusion regimes recovering Maxwell-Stefan model and incompressible Navier-Stokes equations, Phys. Rev. E 80, 056701 (2009).

[17] S. Bennett, P. Asinari, and P. J. Dellar, A lattice Boltzmann model for diffusion of binary gas mixtures that includes diffusion slip, Int. J. Numer. Methods Fluids 69, 171 (2011).

[18] J. Zudrop, K. Masilamani, S. Roller, and P. Asinari, A robust lattice Boltzmann method for parallel simulations of multicomponent flows in complex geometries, Comput. Fluids 153, 20 (2017).

[19] J. Zudrop, S. Roller, and P. Asinari, Lattice Boltzmann scheme for electrolytes by an extended Maxwell-Stefan approach, Phys. Rev. E 89, 053310 (2014).

[20] J. O. Hirschfelder, C. F. Curtiss, and R. B. Bird, Molecular Theory of Gases and Liquids, 2nd ed. (John Wiley \& Sons, New York, 1964).

[21] P. J. A. M. Kerkhof and M. A. M. Geboers, Toward a unified theory of isotropic molecular transport phenomena, AIChE J. 51, 79 (2004).

[22] P. V. Coveney, S. Succi, D. d'Humières, I. Ginzburg, M. Krafczyk, P. Lallemand, and L.-S. Luo, Multiple relaxation time lattice Boltzmann models in three dimensions, Philos. Trans. R. Soc. London A 360, 437 (2002).

[23] S. S. Chikatamarla, S. Ansumali, and I. V. Karlin, Entropic Lattice Boltzmann Models for Hydrodynamics in Three Dimensions, Phys. Rev. Lett. 97, 010201 (2006).

[24] J. Latt and B. Chopard, Lattice Boltzmann method with regularized pre-collision distribution functions, Math. Comput. Sim. 72, 165 (2006).

[25] M. Geier, M. Schönherr, A. Pasquali, and M. Krafczyk, The cumulant lattice Boltzmann equation in three dimensions: Theory and validation, Comput. Math. Appl. 70, 507 (2015).

[26] Z. Guo, C. Zheng, and B. Shi, Discrete lattice effects on the forcing term in the lattice Boltzmann method, Phys. Rev. E 65 , 046308 (2002).

[27] J. C. Maxwell, IV. On the dynamical theory of gases, Philos. Trans. R. Soc. London 157, 49 (1867).

[28] S. Chapman and T. G. Cowling, The Mathematical Theory of Non-uniform Gases, 3rd ed. (Cambridge University Press, Cambridge, 1970).

[29] C. R. Wilke, A viscosity equation for gas mixtures, J. Chem. Phys. 18, 517 (1950).

[30] M. E. McCracken and J. Abraham, Lattice Boltzmann methods for binary mixtures with different molecular weights, Phys. Rev. E 71, 046704 (2005).

[31] N. Looije, J. Gillissen, S. Sundaresan, and H. V. den Akker, Introducing a variable speed of sound in single-component lattice Boltzmann simulations of isothermal fluid flows, Comput. Fluids 167, 129 (2018).

[32] J. M. Buick and J. A. Cosgrove, Investigation of a lattice Boltzmann model with a variable speed of sound, J. Phys. A 39, 13807 (2006).

[33] K. R. Arnold and H. L. Toor, Unsteady diffusion in ternary gas mixtures, AIChE J. 13, 909 (1967).

[34] J. B. Duncan and H. L. Toor, An experimental study of three component gas diffusion, AIChE J. 8, 38 (1962).

[35] R. Taylor and R. Krishna, Multicomponent Mass Transfer (John Wiley \& Sons, New York, 1993)

[36] R. Krishna, Uphill diffusion in multicomponent mixtures, Chem. Soc. Rev. 44, 2812 (2015). 